\title{
Human anti-60 kD heat shock protein autoantibodies are characterized by basic features of natural autoantibodies
}

\author{
Sz Varbiro ${ }^{1}$, A Biro ${ }^{2}$, J Cervenak ${ }^{3}$, L Cervenak ${ }^{4}$, M Singh ${ }^{5}$, F Banhidy ${ }^{1}$, \\ A Sebestyen ${ }^{1}$, G Füst ${ }^{2,4,6}, \mathbf{Z}$ Prohászka ${ }^{2,4,6}$ \\ ${ }^{1} 2^{\text {nd }}$ Department of Obstetrics and Gynecology, Faculty of Medicine, Semmelweis University, Budapest, Hungary \\ ${ }^{2}$ Szentágothai János Knowledge Center, Semmelweis University, Budapest, Hungary \\ ${ }^{3}$ Department of Immunology, Institute of Biology, Eötvös Lóránd University, Budapest, Hungary \\ ${ }^{4}$ Research Group of Inflammation Biology and Immunogenomics, \\ Hungarian Academy of Sciences, Budapest, Hungary \\ ${ }^{5}$ Department of Genome Analysis, Helmholtz Center of Infection Research and Lionex Diagnostics \\ and Therapeutics Ltd, Braunschweig, Germany \\ ${ }^{6} 3^{\text {rd }}$ Department of Medicine, Faculty of Medicine, Semmelweis University, Budapest, Hungary
}

Received: August 11, 2009

Accepted: September 22, 2009

Anti-human Hsp60 autoantibodies - known risk factor of atherosclerosis - were investigated in a mouse model and in samples of healthy subjects: polyreactivity, presence in cord blood samples of healthy newborns and life-long stability were tested. In IgM hybridoma panel from mouse spleens, polyreactivity of anti-Hsp60 autoantibodies was studied. In healthy pregnant women, umbilical vein and maternal blood samples were collected after childbirth, antiHsp-60 and -65 IgM and IgG levels were measured. Life-long stability of anti-Hsp-60 levels was studied on healthy patients during 5 years. ELISA was used in all studies. Polyreactivity of $\operatorname{IgM}$ clones of newborn mice and lifelong stability of these autoantibodies in healthy adults were established. IgM anti-Hsp60 autoantibodies in cord blood of healthy human infants were present, however, there was no correlation between maternal and cord blood IgM anti-Hsp60 concentrations. It is proposed that presence of anti-Hsp60 autoantibodies - as part of the natural autoantibody repertoire - may be an inherited trait. Level of anti-Hsp60 autoantibodies may be an independent, innate risk factor of atherosclerosis for the adulthood.

Keywords: anti-Hsp60 antibodies, atherosclerosis, risk, immune system, pregnancy

Natural antibodies refer to antibodies that are present in the serum of healthy individuals without overt immunization or infection (1). The importance of natural antibodies reacting with self-antigens (natural autoantibodies) received more attention recently (2).

Natural autoantibodies, autoreactive B- and T-cells are present in healthy individuals and are referred to as part of the predominantly selected autoreactive repertoires of the healthy immune system (1).

Heat shock proteins are functionally and immunologically highly conserved molecules present in almost all living organisms $(3,4)$. Bacterial and human Hsp share considerable homology (5) and antibodies or T-cells that recognize microbial Hsp as immunodominant antigens often cross-react with human Hsp (6). Anti-60 kD heat shock protein (Hsp60)

Corresponding author: Szabolcs Varbiro MD, $\mathrm{PhD}$

$2^{\text {nd }}$ Department of Obstetrics and Gynecology

Faculty of Medicine, Semmelweis University

Üllői út 78/A, H-1083 Budapest, Hungary

Phone: +36-20-335-9099; Fax: +36-1-334-6616

E-mail: varbiroszabolcs@gmail.com 
antibodies are indeed present in serum of healthy human subjects $(7,8)$ and also in samples of patients with different diseases incuding atrial fibrillation (9), atherosclerosis (10) and including different forms of vascular diseases (11). The presence of anti-Hsp60 autoantibodies in these diseases is considered as the sign of infection induced autoimmunity (12).

However, van Eden and colleagues (13) and Cohen and c-young (6) formulated alternative hypotheses to explain the presence of self-Hsp reacting antibodies and T-cells. Besides induction through mimicry mechanism, according to their explanation, anti-self Hsp autoreactivity may be the integral part of the normal immune function with a role in selfprotecting, regulating autoimmunity. Basic features of self-protecting, natural autoantibodies include polyreactivity, life-long stability, connectivity, presence in samples of newborn animals without antigenic stimulation (indicating germ-line encoding) and low affinity (14). In humans natural autoantibodies may belong to $\operatorname{IgG}$, IgM and IgA isotypes with the predominance of $\operatorname{IgG}$, whereas in mice IgM autoantibodies dominate $(14,15)$. Previous work indicated that a certain class of autoantibodies, i.e. anti-Hsp autoantibodies, may play significant role in self-protecting autoimmunity (6).

Published evidences indicate important roles for anti-Hsp60 autoantibodies in the pathogenesis of atherosclerosis. The association of elevated anti-Hsp60 levels with different vascular diseases was repeatedly shown (11), and their clinical importance was also indicated by results of prospective studies $(16,17)$. Most importantly, the causal pathogenic role of antiHsp60 antibodies has been shown by Mandal and associates (18) by showing that anti-Hsp60 antibodies purified from sera of patients with documented atherosclerosis when injected into tail vein of apoE deficient mice resulted in accelerated atherosclerosis in them. Some earlier observations of our group, including the description of anti-Hsp60 reactivity independently of common bacterial infections (19); the stability of anti-Hsp60 autoantibody titers over long observation periods (8); and the lack of elevation of anti-Hsp60 IgG in certain diseases characterized by polyclonal B-cell activation (8) supported the view Hsp60 autoantibodies might belong to the group of natural autoantibodies. Therefore, in the present study we aimed at carrying out an in-depth analysis of anti-Hsp60 autoantibodies to investigate whether they carry some characteristics of natural autoantibodies and whether they are part of the innate immune system. To achieve this goal the following parameters were investigated: polyreactivity and presence in cord blood samples of healthy newborns.

\section{Methods}

\section{Mouse IgM hybridoma panel}

The establishment of the IgM hybridoma panel from spleens of healthy newborn $\mathrm{balb} / \mathrm{c}$ mice consisting of 26 different clones was described earlier (20). Supernatants stored at $-20^{\circ} \mathrm{C}$ were used in our experiments. The investigation conforms with the Guide for the Care and Use of Laboratory Animals published by the US National Institutes of Health.

\section{Maternal and cord blood samples}

Following informed consent 51 healthy pregnant women after complication-free pregnancies took part in the study, which was approved by the Ethical Committee and was carried out in accordance with the Declaration of Helsinki. The mean age of the mothers at delivery was 28 years (range, 20-41 yrs). Sera were separated from two blood samples, one taken 
from the placental part of the umbilical vein and the other taken from the antecubital vein of mothers within 12 hours after complication-free childbirth, and were stored at $-70^{\circ} \mathrm{C}$ until processing.

\section{Healthy adult men}

The Second Northwick Park Heart Study has been described previously (21). The 4600 men 50-61 years registered in 9 medical practices in Great Britain were screened for eligibility for the study. Exclusion criteria were: a history of unstable angina or acute myocardial infarction (AMI); a major Q wave on the electrocardiogram (ECG); regular anti-platelet or anticoagulant therapy; cerebrovascular disease; life-threatening malignancy; history of hepatitis B or other infectious disease; diabetes; high blood pressure (BP); kidney and liver problems; high cholesterol or thyroid problems at baseline; angina pectoris at any follow up, or precluding informed consent. Forty-eight from those who remained disease-free during follow-up (5 years) were randomly selected for this study and stored serum samples obtained in three points of time (baseline, after 3 years and after 5 years) were provided.

\section{Analytical measurements}

The amounts of IgG-type antibodies reacting with proteins of the Hsp 60 family [recombinant human hsp60 (StressGen, SPP-740, Victoria, Canada); recombinant M. bovis hsp65 (batch MA14, GBF, Braunschweig, Germany, supported by the UNDP/World Bank/WHO Special Program for Research and Training in Tropical Diseases)] were assessed by ELISA as described previously (8).

In brief, plates were coated with $0.1 \mu \mathrm{g} /$ well human hsp60 or M. bovis hsp65. After washing and blocking (PBS, $0.5 \%$ gelatin) the wells were incubated with $100 \mu \mathrm{L}$ of serum samples diluted $1: 500$ in PBS containing $0.5 \%$ gelatin and $0.05 \%$ Tween 20 . Binding of antihsp antibodies was determined using $\gamma$-chain specific anti-human IgG peroxidase labeled antibodies (Sigma, St. Louis, USA) and an O-phenylene-diamine (SIGMA) detection system. The optical density was measured at $490 \mathrm{~nm}$ (reference at $620 \mathrm{~nm}$ ) and the means of duplicate wells were calculated after subtraction of OD registered in uncoated wells. A serial dilution of a high-reacting human serum was used as standard. Data obtained as optical density values were calculated to arbitrary unit $/ \mathrm{mL}$ values related to this standard.

Measurement of IgM type anti-hsp60 was done on plates coated with $5 \mu \mathrm{g} / \mathrm{ml}$ human hsp60 or M. Bovis Hsp65. Incubation with the sample was done at $4{ }^{\circ} \mathrm{C}$ over night, and the wells were developed with rabbit anti-human-IgM-POX and anti-rabbit-IgG-POX-conjugates together to amplify the specific reaction. Since there were no available high-titer serum samples to be used as standard results are indicated as OD values. Each measurement was done on one particular day, using two ELISA plates, and there was no significant plate to plate variability thus there was no need to normalize the data. Means of duplicate measurements were calculated after subtraction of background. Samples of adults or newborns were diluted 1:400 or 1:20, respectively, in the anti-Hsp60/65 assays.

Total IgG and IgM levels were determined by sandwich-type ELISA. Plates were coated with mouse anti-human IgG or IgM antibodies, incubated with test samples and developed with rabbit anti-human IgG and IgM peroxidase conjugates following incubation. Purified human $\operatorname{IgG}$ and $\operatorname{IgM}$ were used as standards. 


\section{Statistical analysis}

As the variable antibody to heat shock proteins was not normally distributed, non-parametric tests were used for group comparisons, and Spearman rank correlation coefficients were calculated for estimating interrelations between anti-Hsp antibodies and other variables. The significance level was set at the value of $p<0.05$. Non-parametric values are presented as median $(25$ th -75 th percentile), parametric values as mean \pm standard deviation.

\section{Results}

\section{Polyreactivity of anti-Hsp60 autoantibodies}

Twenty-six IgM hybridoma clones from newborn healthy mice were tested for their reactivity against Hsp60. These clones have earlier been tested for their reactivity towards haptens and common autoantigens (20). Table I presents the results of our Hsp60 reactivity measurements together with previous findings. Five out of the 26 clones were shown to react with human Hsp60 and these clones were reactive against at least 4 antigens out of the 6 investigated. Two out of these 5 clones were even reacting with all the 6 antigens tested. It is important to note, that there are no sequence homologies or functional similarities between the analyzed antigens and Hsp60 (two antigens belong to haptens).

\section{Presence of IgM anti-Hsp60 antibodies in cord blood samples of healthy newborns}

Blood samples of 51 healthy newborns and their mothers were investigated for the presence of IgG and IgM type antibodies reacting with human and M. bovis Hsp60-family proteins in this ethical-committee approved consecutive study. The median age of the mothers was $28 \pm 4.8( \pm \mathrm{SD})$ years (range 20-41 years). Only women with complication-free pregnancies were included in our study; $45 \%$ of the population never smoked, $42 \%$ smoked beforehand, and $13 \%$ smoked during pregnancy. Mothers gave birth to healthy, mature newborns (each singlet, $51 \%$ girls).

Results of anti-Hsp60 and anti-Hsp65 measurements are presented in Figure 1. Median of total IgG concentration in the maternal samples was $8.17 \mathrm{~g} / \mathrm{l}$ (IQ range 6.7-9.54) and in cord blood samples $9.93 \mathrm{~g} / 1$ (8.61-11.8). Total IgM levels were as follows: $1.56 \mathrm{~g} / 1$ (0.862.2 , maternal samples) and $0.073 \mathrm{~g} / 1$ (0.056-0.103, for newborns). These concentrations fall within the age-adjusted reference ranges of the two groups.

Both IgG and IgM anti-Hsp60 specific autoantibodies were present in all cord blood and maternal samples. In contrast, only IgG anti-Hsp65 antibodies were detected in all samples; IgM anti-Hsp65 antibodies were absent from maternal samples and only 9/51 (18\%) of the cord blood samples showed reactivity towards Hsp65. Whereas highly significant correlation between maternal and cord blood IgG anti-Hsp60 levels was observed ( $\mathrm{r}=0.901$; $\mathrm{p}<0.0001$ ), there was no similar correlation between maternal and cord blood IgM anti-Hsp60 concentrations $(r=0.03 ; p=0.823)$. 
Table I Reactivity pattern of an IgM monoclonal antibody panel of healthy newborn mice with certain haptens and antigens*

\begin{tabular}{|c|c|c|c|c|c|c|c|}
\hline Clone & TNP-BSA & FITC-BSA & BSA & DNS & Actin & Hsp60 & \# of positive \\
\hline $1 / 1$ & & & & & & & 3 \\
\hline $1 / 4$ & & & & & & & 3 \\
\hline $1 / 5$ & & & & & & & 4 \\
\hline $1 / 11$ & & & & & & & 3 \\
\hline $2 / 3$ & & & & & & & 5 \\
\hline $2 / 5$ & & & & & & & 3 \\
\hline $2 / 13$ & & & & & & & 4 \\
\hline $2 / 15$ & & & & & & & 3 \\
\hline $2 / 21$ & & & & & & & 4 \\
\hline $3 / 9$ & & & & & & & 4 \\
\hline $3 / 17$ & & & & & & & 5 \\
\hline $3 / 25$ & & & & & & & 2 \\
\hline $4 / 5$ & & & & & & & 2 \\
\hline $4 / 6$ & & & & & & & 4 \\
\hline $4 / 12$ & & & & & & & 2 \\
\hline $4 / 20$ & & & & & & & 1 \\
\hline $4 / 21$ & & & & & & & 6 \\
\hline $6 / 17$ & & & & & & & 2 \\
\hline $6 / 26$ & & & & & & & 4 \\
\hline $6 / 34$ & & & & & & & 5 \\
\hline $7 / 1$ & & & & & & & 2 \\
\hline $7 / 8$ & & & & & & & 3 \\
\hline $7 / 16$ & & & & & & & 4 \\
\hline $7 / 20$ & & & & & & & 5 \\
\hline $8 / 8$ & & & & & & & 6 \\
\hline $8 / 14$ & & & & & & & 4 \\
\hline
\end{tabular}

* The antibody reactivity is indicated by the highest dilution giving an OD $>0.1$ on the gray-scale; black: 1:400; dark gray 1:100; gray 1:20; white: $\mathrm{OD}<0.1$ in all dilutions. TNP: 2,4,6 trinitrophenyl; FITC: fluorescein isothyocianate, BSA: bovin serum albumin

Table II Changes of anti-Hsp60 autoantibody levels during 5 years of follow up in healthy men

\begin{tabular}{|l|c|c|c|}
\hline \multirow{2}{*}{} & Baseline & Third year & Fifth year \\
\cline { 2 - 4 } & \multicolumn{3}{|c|}{ Sample } \\
\hline Anti-Hsp60 (AU/ml, n=48) & $62.4(32.9-99.5)^{*}$ & $53.5(35.2-84.3)^{*}$ & $51.6(31.8-97.1)^{*}$ \\
\hline Correlation to third year's sample & $0.806(<0.0001)^{* *}$ & - & - \\
\hline Correlation to fifth year's sample & $0.817(<0.0001)^{* *}$ & $0.845(<0.0001)^{* *}$ & - \\
\hline
\end{tabular}

* median (interquartile range);

** Spearman rank correlation coefficient (p) 

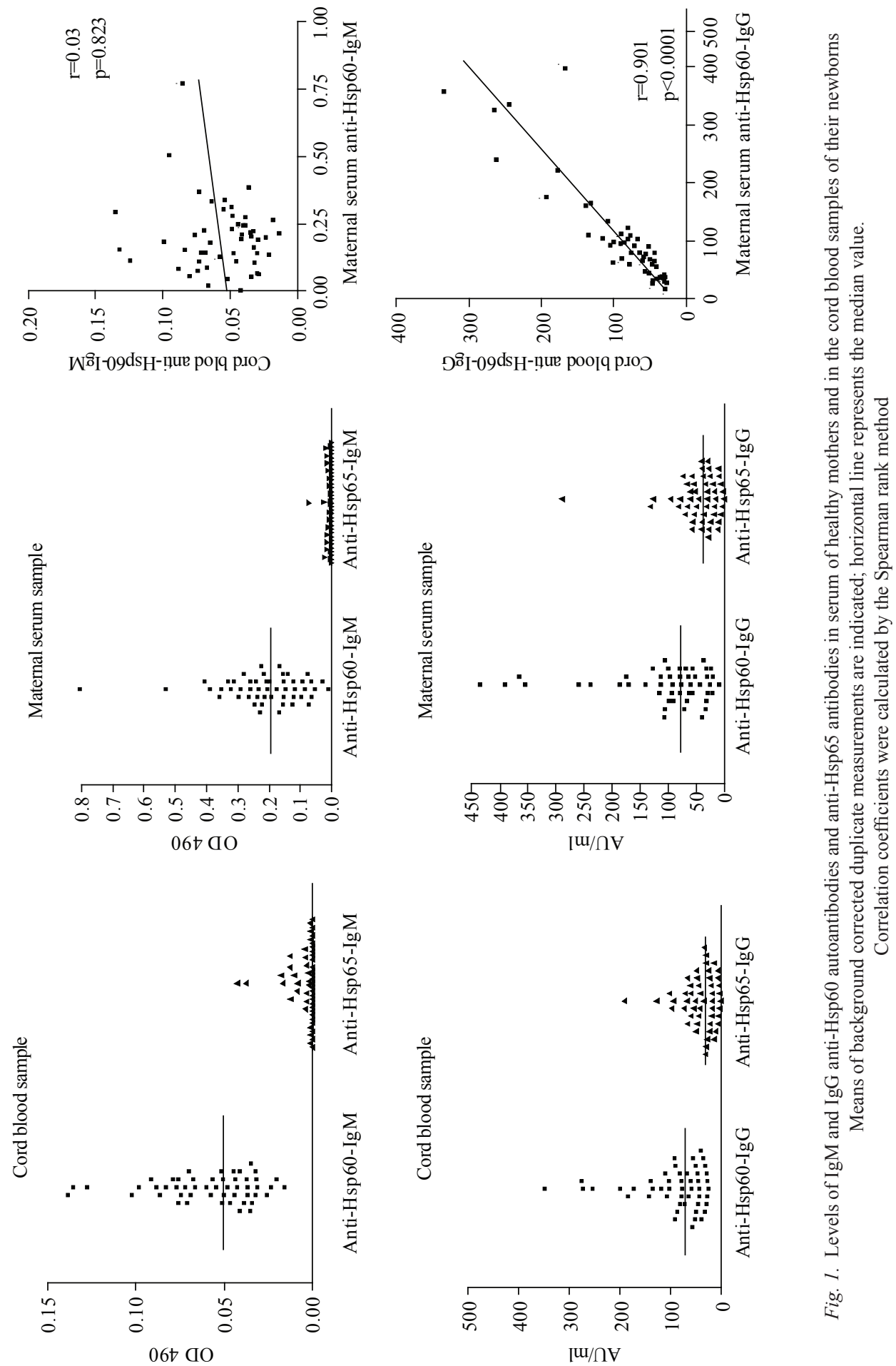
Forty-eight subjects recruited into the Second Northwick Park Heart Study who remained disease free during follow-up with available stored samples were included into our study. Baseline and two follow-up serum samples (years 3 and 5) were studied for the presence of specific anti-Hsp60 IgG antibodies. As presented in Table II, median anti-Hsp60 level in the baseline sample was $62.4 \mathrm{AU} / \mathrm{ml}$ and this level did not significantly change during the follow up (53.5 and 51.6 AU/ml, respectively in years 3 and $5 ; \mathrm{p}=0.44$, Friedman test). There were highly significant correlations between autoantibody levels measured in the three points of time (Table II). Figure 2 shows antibody levels in subgroups of subjects stratified into quartiles according to baseline concentrations. As presented, those who belong to the low, respectively, high concentration groups at the start of the study belong to the same groups at the end of the follow-up. There are no changes or crossings between the groups during follow up ( $p>0.05$ for each of the subgroups).

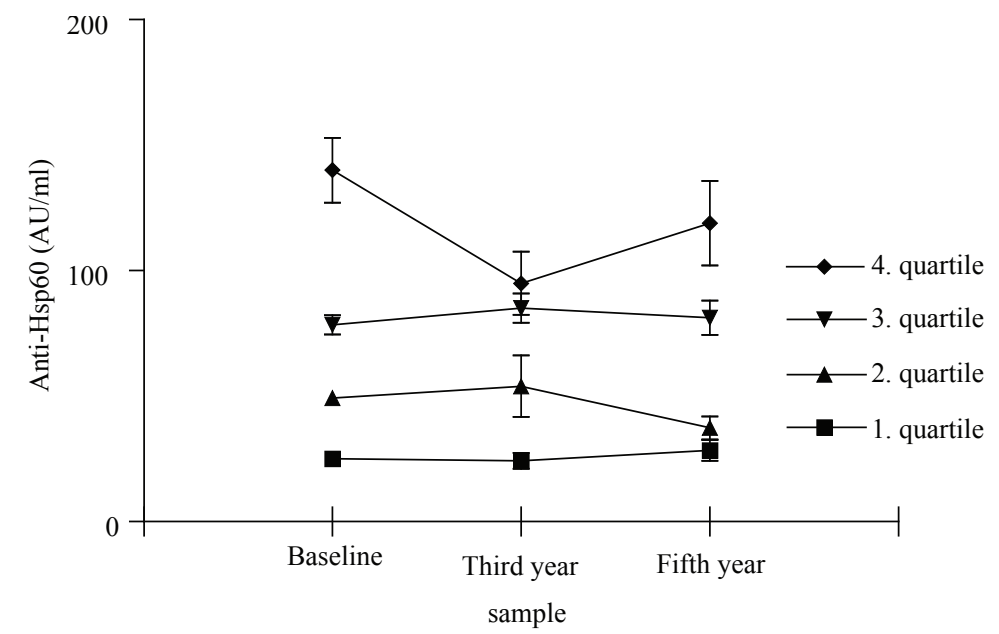

Fig. 2. Changes in anti-Hsp60 autoantibody levels in healthy men during five years of follow-up Data are presented as means with SEM of the groups

\section{Discussion}

In this study anti-human Hsp60 autoantibodies were investigated in a mouse IgM hybridoma panel and in samples of healthy subjects whether they bear some characteristics of natural autoantibodies. Polyreactivity of IgM clones of newborn mice, presence of IgM anti-Hsp60 autoantibodies in cord blood of healthy human infants and life-long stability of these autoantibodies in healthy adults were investigated. All of these traits are characteristic for anti-Hsp60 autoantibodies as shown here for the first time.

The first novel observation of this study is that a panel of unselected polyreactive IgM antibodies of newborn healthy mice contains antibodies reacting with Hsp60 as well. This panel was investigated for its reactivity against BSA, actin, DNA and different haptens, and the vast majority of the clones have been shown to be polyreactive (20). Specific reactivity towards Hsp60 was, however, not examined for these or other natural autoantibodies earlier. Importantly, those IgM antibodies showing reactivities against at least 4 antigens or haptens 
were found to be reactive against Hsp60. Furthermore, Pashov and co-workers revealed evidence that actin-purified polyreactive autoantibodies representing the natural autoantibody repertoire, also reacted with Hsp90 (22). Thus, our novel observations and the literature data support the conclusion that natural autoantibodies in mice contain anti-Hsp60 antibodies and these are characterized by polyreactivity.

The second novel observation of the present study is the description of antibodies against human and M. bovis $60 \mathrm{kDa}$ Hsp in cord blood samples of healthy newborns and in their mothers. In all of the investigated cord blood samples the presence of significant amounts of IgM anti-Hsp60 antibodies was shown. Since none of the babies or their mothers suffered from any illness, these antibodies may be considered as natural autoantibodies. Notably is the fact that levels of IgM anti-Hsp60 did not correlate to maternal IgM anti-Hsp60 concentrations. Since IgM antibodies do not cross the placenta in human this observation strongly supports our conclusion that the $\operatorname{IgM}$ repertoire of healthy human newborns contains anti-Hsp60 antibodies and this immunological compartment is independent from the mother's IgM pool. Anti-Hsp65 IgM antibodies were present in much lower titers in the cord blood samples and were absent from maternal sera. Although high level of amino-acid similarity exists between Hsp60 and Hsp65 convincing evidence suggest that important differences are also present in their epitope structure $(23,24)$. Antibodies reacting with Hsp65 represent mainly serological signs of different infections and are induced by molecular mimicry (25). Thus, our results on the lack of significant anti-Hsp65 IgM in mothers confirm the proper patient selection process including complication-free pregnancies only. The IgG anti-Hsp60 pool may also contain natural autoantibodies both in the mother and in the newborn, however, since IgG cross the placenta, the independent nature of the infant and maternal immunological compartments cannot be investigated in these samples.

According to the third aim of the study changes in anti-Hsp60 levels over a long observation period was investigated. A large collection of control serum samples from the Second Northwick Park Heart study was applied for this experiment. Anti-Hsp60 IgG levels were correlating in three different time points measured during the 5-year long followup period. Those who had low, respectively, high anti-Hsp60 IgG levels in the first year remained in the same strata at the end of the observation period. This observation indicates tight regulation of IgG anti-Hsp60 autoantibody levels in healthy adults which is in complete accordance with our earlier results indicating independence of these autoantibodies from serological signs of different infections (19). Our results document the stability of IgG antibody reactivity directed toward the conserved Hsp60 through an observation period of five years. Similarly, Lacroix-Desmazes et al. (15) observed stability of the natural selfreactive IgM and IgG antibody repertoires with aging supporting the view that autoreactive B cells in the normal immune system are positively selected for reactivity with a limited set of immunodominant self-antigens throughout life. Our results indicate that Hsp60 also belong to this limited set of dominant self-antigens. Although autoantibody levels against conserved proteins remained stable with age, large inter-individual differences were observed with regard to reactivities (26). Our data indicate that IgG reactivity toward Hsp60 may also be characteristic for a given individual as well, which is consistent with the findings of LacroixDesmazes et al. (14) about the specificity of self-reactive antibody repertoire and with the concept of "antibody finger-printing" initially proposed by Francoeur (27).

Based on our earlier and current results together with literature data we propose that atherosclerosis associated anti-Hsp60 antibodies may originate from two strongly related sources. First, multiple and repeated infections may induce anti-Hsp60 antibodies cross reacting between microbial and human antigens. Second, anti-Hsp60 antibodies are present in 
the natural autoantibody repertoire as well and the size and quality of this repertoire is under control of genetic and environmental factors. The environmental control is mainly related to the cumulative effect of infections and this is why the two sources of anti-Hsp60 antibodies are strongly related. The size of the repertoire, i.e. the large inter-individual differences, is probably under the strict control and regulation of genetic factors. Our first observation obtained in a healthy Finnish population identified promoter polymorphism of interleukin- 6 as one of the underlying regulatory factors (17), and these results were confirmed in a recent independent study (28). Furthermore, epistatic interaction between immunoglobulin GM allotypes and interleukin-6 was also identified in association with increased anti-Hsp60 antibody levels.

Therefore we suggest that the carriage of high anti-Hsp60 autoantibody levels (as part of the natural autoantibody repertoire) may be an inherited trait and the cumulative antibody inducing effects of multiple infections add to this trait. After understanding this relationship we will be able to estimate atherosclerosis risk early, in neonates or during childhood.

\section{Acknowledgements}

This work was supported by research grants from the Hungarian Scientific Research Fund (T 046837) from the Ministry of Health (ETT 229/2006) and by the Bolyai Research Fellowship (BO/00080/2003), research grant of the Hungarian Society of Hypertension.

The skilful technical assistance of Szigeti Antalné and Margit Kovács is acknowledged with many thanks. We are highly indebted to Dr. George Miller who provided the NPHSII serum samples.

\section{REFERENCES}

1. Coutinho A, Kazatchkine MD, Avrameas S: Natural autoantibodies: Curr. Opin. Immunol. 7, 812-818 (1995)

2. Lacroix-Desmazes S, Kaveri SV, Mouthon L, Ayouba A, Malanchere E, Coutinho A, Kazatchkine MD: Selfreactive antibodies (natural autoantibodies) in healthy individuals. J. Immunol. Methods 216, 117-137 (1998)

3. Ellis RJ.: Protein misassembly: macromolecular crowding and molecular chaperones. Adv. Exp. Med. Biol. 594, $1-13$ (2007)

4. Lamb DJ, El-Sankary W, Ferns GA: Molecular mimicry in atherosclerosis: a role for heat shock proteins in immunisation. Atherosclerosis 167, 177-185 (2003)

5. Jones DB, Coulson AFW, Duff GW: Sequence homologies between Hsp60 and autoantigens. Immunol. Today 14, 115-118 (1993)

6. Cohen IR, Young DB: Autoimmunity, microbial immunity and the immunological homunculus. Immunol. Today 12, 105-110 (1991)

7. Pockley AG, Blumer J, Hanks BM, Wright BH: Identification of human heat shock protein 60 (Hsp60) and antiHsp60 antibodies in the peripheral circulation of normal individuals. Cell Stress Chap. 4, 29-35 (1999)

8. Prohaszka Z, Duba J, Horvath L, Csaszar A, Karadi I, Szebeni A, Singh M, Fekete B, Romics L, Fust G: Comparative study on antibodies to human and bacterial $60 \mathrm{kDa}$ heat shock proteins in a large cohort of patients with coronary heart disease and healthy subjects. Eur. J. Clin. Invest. 31, 285-292 (2001)

9. Mandal K, Jahangiri M, Mukhin M, Poloniecki J, Camm AJ, Xu Q: Association of anti-heat shock protein 65 antibodies with development of postoperative atrial fibrillation. Circulation 110, 2588-2590 (2004)

10. Ghayour-Mobarhan M, Lamb DJ, Tavallaie S, Ferns GA: Relationship between plasma cholesterol, von Willebrand factor concentrations, extent of atherosclerosis and antibody titres to heat shock proteins-60, -65 and -70 in cholesterol-fed rabbits. Int. J. Exp. Pathol. 88, 249-255 (2007)

11. Wick G, Knoflach M, Kind M, Henderson B, Bernhard D: Heat shock proteins and stress in atherosclerosis. Autoimmun. Rev. 3(Suppl 1), S30-31 (2004)

12. Mayr M, Xu Q, Wick G: Atherogenic effects of chronic infections: the role of heat shock protein 60 in autoimmunity. Isr. Med. Assoc J. 1, 272-277 (1999)

13. van Eden W, Koets A, van Kooten P, Prakken B, van der Zee R: Immunopotentiating heat shock proteins: negotiators between danger and control of autoimmunity. Vaccine. 21, 897-901 (2003)

14. Lacroix-Desmazes S, Mouthon L, Coutinho A, Kazatchkine MD: Analysis of the natural human IgG antibody 
repertoire: life-long stability of reactivities toward self antigens contrasts with age-dependent diversification of reactivities against bacterial antigens. Eur. J. Immunol. 25, 2598-2604 (1995)

15. Lacroix-Desmazes S, Mouthon L, Kaver SV, Kazatchkine MD, Weksler ME: Stability of natural self-reactive antibody repertoires during aging. J. Clin. Immunol. 19, 26-34 (1999)

16. Huittinen T, Leinonen M, Tenkanen L, Manttari M, Virkkunen H, Pitkanen T, Wahlstrom E, Palosuo T, Manninen V, Saikku P: Autoimmunity to human heat shock protein 60, Chlamydia pneumoniae infection, and inflammation in predicting coronary risk. Arterioscler. Thromb. Vasc. Biol. 22, 431-437 (2002)

17. Veres A, Fust G, Smieja M, et Heart Outcomes Prevention Evaluation (HOPE) Study Investigators: Relationship of anti-60 kDa heat shock protein and anti-cholesterol antibodies to cardiovascular events. Circulation 106, 2775-2780 (2002)

18. Mandal K, Jahangiri M, Mukhin M, Poloniecki J, Camm AJ, Xu Q: Association of anti-heat shock protein 65 antibodies with development of postoperative atrial fibrillation. Circulation 110, 2588-2590 (2004)

19. Burian K, Kis Z, Virok D, Endresz V, Prohaszka Z, Duba J, Berencsi K, Boda K, Horvath L, Romics L, Fust G, Gonczol E: Independent and joint effects of antibodies to human heat-shock protein 60 and Chlamydia pneumoniae infection in the development of coronary atherosclerosis. Circulation 103, 1503-1508 (2001)

20. Cervanak J, Kiss K, Uher F: Partial characterization of two lymphocyte-specific natural autoantibodies isolated from newborn mice. Acta Microbiol. Immunol. Hung. 46, 53-62 (1999)

21. Miller GJ, Bauer KA, Barzegar S, Cooper JA, Rosenberg RD: Increased activation of the haemostatic system in men at high risk for fatal coronary heart disease. Thromb. Haemost. 75, 767-771 (1996)

22. Pashov A, Kenderov A, Kyurkchiev S, Kenderov A, Kyurkchiev S, Kehayov I, Hristova S, Lacroix-Desmazes S, Giltiay N, Varamballi S, Kazatchkine MD, Kaveri SV: Autoantibodies to heat shock protein 90 in the human natural antibody repertoire. Int. Immunol. 14, 453-461 (2002)

23. Prohaszka Z, Duba J, Lakos G, Kiss E, Varga L, Janoskuti L, Csaszar A, Karadi I, Nagy K, Singh M, Romics L, Fust G: Antibodies against human heat-shock protein (hsp) 60 and mycobacterial hsp65 differ in their antigen specificity and complement-activating ability. Int. Immunol. 11, 1363-1370 (1999)

24. Uray K, Hudecz F, Fust G, Prohaszka Z: Comparative analysis of linear antibody epitopes on human and mycobacterial $60-\mathrm{kDa}$ heat shock proteins using samples of healthy blood donors. Int. Immunol. 15, 1229-1236 (2003)

25. Wick G, Knoflach M, Xu Q: Autoimmune and inflammatory mechanisms in atherosclerosis. Annu. Rev. Immunol. 22, 361-403 (2004)

26. Mouthon L, Lacroix-Desmazes S, Nobrega A, Barreau C, Coutinho A, Kazatchkine MD: The self-reactive antibody repertoire of normal human serum $\operatorname{IgM}$ is acquired in early childhood and remains conserved throughout life. Scand. J. Immunol. 44, 243-251 (1996)

27. Francoeur AM: Biotechnology in forensic medicine: new ways of fingerprinting. J. Biotechnol. 10, 203-208 (1989)

28. Kiszel P, Fust G, Pessi T, Hurme M, Prohaszka Z: Associations between interleukin-6 genetic polymorphisms and levels of autoantibodies to 60-kDa heat-shock proteins. Hum. Hered. 62, 77-83 (2006) 